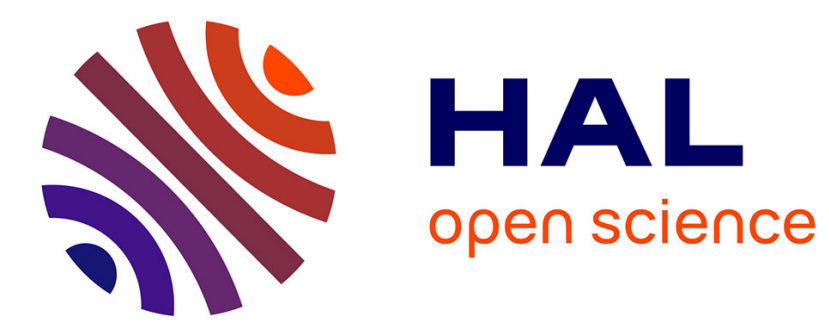

\title{
Editorial overview: Developmental neuroscience
}

Denis Jabaudon, Alain Chédotal

\section{To cite this version:}

Denis Jabaudon, Alain Chédotal. Editorial overview: Developmental neuroscience. Current Opinion in Neurobiology, 2021, 66, pp.iii-v. 10.1016/j.conb.2021.03.006 . hal-03206671

\section{HAL Id: hal-03206671 \\ https: / hal.sorbonne-universite.fr/hal-03206671}

Submitted on 23 Apr 2021

HAL is a multi-disciplinary open access archive for the deposit and dissemination of scientific research documents, whether they are published or not. The documents may come from teaching and research institutions in France or abroad, or from public or private research centers.
L'archive ouverte pluridisciplinaire HAL, est destinée au dépôt et à la diffusion de documents scientifiques de niveau recherche, publiés ou non, émanant des établissements d'enseignement et de recherche français ou étrangers, des laboratoires publics ou privés. 


\section{Editorial overview: Developmental neuroscience}

The past few years have seen an explosion in new technologies that have brought forward our understanding of the development of the central nervous system at the single-cell level. Singlecell RNA sequencing has shed a crisp light on the molecular diversity of cell types, while advanced 3D imaging techniques such as light-sheet microscopy now allow single axons to be identified and followed in intact cleared brains, bringing a new understanding on the assembly of cells into circuits and networks during development. Our genetic toolbox has been expanded by the development of new molecular tools allowing targeted ablation or silencing of single genes in defined cell types, and incremental progress in the generation and culture of 3D brainlike organoids and assembloids have informed our understanding of normal and abnormal developmental processes in humans.

This volume of Current Opinion in Neurobiology is meant to exemplify some of these developmental facets, and contrast and unite them. Investigators at the forefront of their fields provide us with a state-of-the-art view of their topic of research, with at times related topics covered by multiple authors to provide a community point-of-view on complex issues.

How new technologies that can help understanding nervous system development are specifically covered in several articles. Shi and colleagues describe the methods and strategies to make human brain organoids and the role of self-organization (intrinsic) and extrinsic factors (growth factors, morphogens, extracellular matrix) in this process. Organoids can provide important insights into understanding human brain development, neurodevelopmental disorders and brain evolution. However, several challenges still lie ahead like the limited gyration, the low cellular diversity and the lack of vascularization. Le Bail and colleagues also tackle these questions in terms of temporal specification: how diverse are cortical neurons and what are the factors controlling the maintenance of cortical progenitors in vivo and in organoids. They next illustrate what these in vitro models have brought to our knowledge of human brain evolution and the unique features of human cortex development. Nedelec and Martinez-Arias also address these questions but taking the spinal cord as a model. After giving an overview of the molecular mechanisms that control spinal cord patterning and the specification of motor neurons and interneurons, they explain how this knowledge has been used to orient the in vitro differentiation of human pluripotent stem cells into spinal cord neurons, Again, generating the full spectrum of motor neuron diversity remains an important issue. Building locomotor circuits in a dish seems to be within reach which should help understanding the etiology of diseases such as ALS and developing new therapeutics for neuromuscular diseases. Also focusing on human brain organoids, Reiner and colleagues review on how these 3D structures can help us identify and understand human-specific processes of migration during development. emphasizing the value of such approaches in studying the pathophysiological bases of cortical malformations such as Miller-Dieker syndrome or lissencephaly. A final technology-oriented article is the one by Solanelles-Farre and Telley, who review the latest applications to the field of developmental neurobiology of multi-omics technologies such as single-cell RNA sequencing (scRNAseq) and single-cell assay for transposase (scATASseq) provide a compendium of developmental studies in this field to date. Cell profiling techniques, can unravel the transcriptome and epigenome of any cell type and significantly extended the cell repertoires of the brain and spinal cord. Lineage 
studies, using scRNAseq or genetic barcoding (somatic mutations) have also revealed unknow features of early brain patterning or cortical neuron specification.

In addition to these techniques-oriented reports, a variety of biology-centered reviews highlight multiple facets of brain development, from progenitor biology to neurogenesis, migration, axonal guidance and synaptogenesis.

The complex and emerging question of neuronal lineages in the developing cortex is covered by Lin and colleagues and Llorca and Marin, with a particular focus on how many types of progenitors are required to generate the diverse types of neurons present in the adult. An array of models has been proposed from strictly deterministic (i.e. single neuron types emerge from corresponding single progenitor types) to fully stochastic, whereby neurons emerge somewhat randomly (albeit with a temporal bias) from multicompetent progenitors as corticogenesis unfolds. Fischer and Morin also address the question of progenitor competence with a particular focus on "molecular recorders", which are molecular devices which can in principle count cell divisions or record progressing molecular identities. Finally, Bonnefont and Vanderhaeghen examine the question of temporality in progenitor competence and neuron differentiation, highlighting species-specific developmental paces and critical periods of plasticity. Molecular mechanisms controlling cell fate is discussed by Ortiz-Alvarez and Spassky while Hoye and Silver specifically address translational controls over cell fate, in particular with regard to translation in the basal processes of apical radial glia.

Abnormal neuronal migration can alter cortical size, layering and folding leading to various neurological disorders. Castello and Gleeson review malformations of cortical development such as lissencephaly, epilepsy, tuberous sclerosis (TSC) and their current therapies. Biallelic mutations of new culprit genes have been recently identified, in Reelin, several genes encoding component of the extracellular matrix such as laminins and collagens or enzymes (such as TMRC3) controlling protein glycosylation. But in addition, there is mounting evidence suggesting that more focal cortical dysplasia and heterotopia, such as TSC can be attributed to the combination of germline and somatic mutations which are more difficult to mimic in animal models. The relevance of mouse models or human cortical malformations is also discussed by Francis and Capello who explore the underlying molecular mechanisms with a focus on the cytoskeleton and centrosome. Tubulinopathies comprise a large and heterogeneous range of brain disorders associated with mutations in tubulins, associated proteins and motor proteins. The authors describe the novel mutations identified in kinesins, dyneins and a dynein adaptor or centrosomal components and how, by affecting cytoskeleton dynamics, they perturb neuronal progenitor proliferation and survival in addition to cell migration. They also emphasize the variability in the severity of these malformations across the cortex which remains to be understood.

Although neuronal migration is primarily a developmental process, it persists in the rostral migratory stream of rodents, along which neuroblasts migrate from the subventricular zone (SVZ) to the olfactory bulb. Nakajima and colleagues report on the mechanisms that orient the migration of SVZ-derived neuroblasts and the influence of extrinsic factors produced by astrocytes, serotoninergic inputs, the vasculature and choroid plexus. Evidence supporting a conservation of postnatal neuroblast migration in humans, a vivid debate, is also presented. In a last part, the authors discuss recent studies showing that in various models of brain injury, SVZ-derived cells can migrate to the lesions and how the use of biomaterials could facilitate the recruitment of neuroblasts and brain repair.

Increasingly, molecules whose function was thought to be largely elucidated find themselves "repurposed" for other developmental processes, and novel axon guidance molecules emerge in addition to the "canonical" ones. 
In their chapter, Guy and Kamiguchi review the unconventional role of lipids in axon guidance taking as examples Lysophosphatidic acid in the barrel cortex, sphingosine-1 phosphate and Lysophosphatidylinositol in the visual system and Lysophosphatidylglucoside in the spinal cord. Although some of their receptors have been identified, their regulation, mechanisms of action and signaling pathways in growth cones are still largely unknown. The current limitations and technical complexity of lipidomics studies is also highlighted by the authors. Growth factors, such as VEGF, HGF, GDNF or FGF are best known for modulating cell survival and proliferation but in their chapter, Short and colleagues provide a perspective on their role in midline crossing, axon pathfinding in the peripheral nervous system and visual system. They also report on growth factor control of neuronal migration and synaptogenesis. The pleiotropy of molecules controlling brain development is also highlighted by Faini and colleagues who present the non-canonical roles of Reelin in axonal guidance and by Limoni and Niquille, who review the diverse roles played by semaphorins and their receptors in neurogenesis, neuronal migration and oligodendrocyte development. Last, the chapter by Dorskind and Kolodkin describe recent findings that have provided novel and surprising insights into the mechanism of action of netrin-1 and DCC in axon guidance, the regulation of axon responsiveness to midline-derived cues, and the discovery of novel axon guidance molecules such as teneurins and DPRs.

Marking the transition between pre-natal and post-natal development, Gesuita and Karayannis and Genescu and Garel provide us with their view on cortical layer 1, and on how external inputs act there to sculpt circuit structure and function. The link between development and circuits is also provided by Kennedy and colleagues, who take meso-scale approaches to discuss circuit organization and Artur Kania, who provides his perspective on the development and plasticity of the somatosensory cortex. Circuit assembly and function are further discussed from a multitude of points of view: Hoersting and Schmucker discuss mounting evidence suggesting that neuropsychiatric diseases such as schizophrenia have a neurodevelopmental origin and might be related to abnormal neurite arborization. Understanding the underlying mechanisms requires the development of improved imaging techniques to access neuronal morphology and its heterogeneity at a single cell resolution. Next, they summarize the molecules and pathways that orchestrate the development of axonal and dendritic arborization and neuronal shape. In their review, Blockus and Polleux provide an integrative view on circuit assembly, with a particular focus on the hippocampus where they discuss combinatorial vs. unitary codes underlying synaptic specificity. Finally, Fossati and Charrier discuss recent work on the unexpected role of ionotropic glutamate receptors in synaptogenesis in the cerebellum, hippocampus and cortex, via a promotion of trans-synaptic interactions.

Together, these reviews address most of the outstanding questions on circuit formation. It shows how new technologies and a multidisciplinary approach should help answering them. It also illustrates the diversity of animal models, neuronal systems and molecules that are studied in this very active research field.

\section{Denis Jabaudon}

Dept. of Basic Neurosciences, University of Geneva, Switzerland e-mail: denis.jabaudon@unige.ch

Denis Jabaudon is currently a professor at the University of Geneva, the Director of the Geneva University Neurocenter, and also practices as a clinical neurologist at Geneva University Hospital. His work is funded by the Swiss National Science Foundation and the European Research Council (Advanced grant). Pr. Jabaudon's research focuses on 
understanding how genetic and input-dependent mechanisms interact to control neuronal circuit assembly during cortical development. His laboratory has identified key mechanisms underlying the generation of neuronal diversity during corticogenesis, including showing that electric properties of cortical progenitors are instrumental to generate different types of neurons, identifying sequential molecular steps involved in cortical neuron generation, and revealing that cortical progenitors retain the ability to generate normally previously-born neurons. Denis Jabaudon is FENS KAVLI network of excellence alumnus and his research has earned him several awards including the Cloetta prize and Robert Bing Prize .

\section{Alain Chédotal \\ Institut de la Vision, INSERM, Sorbonne Université, Paris \\ Email: alain.chedotal@inserm.fr}

Alain Chédotal, is research director at the French National Institute for Health and Medical Research (INSERM) and group leader at the Institut de la Vision in Paris. He was an undergrad at the Ecole Normale Supérieure de Lyon, received a PhD in Neuroscience from Pierre \& Marie Curie University in Paris and conducted a postdoctoral research at The University of California at Berkeley. Dr Chédotal was elected at the French Academy of Sciences, the Academia Europaea and the European Molecular Biology Organization. He is a member of the European Dana alliance for Brain Initiatives. Dr Chédotal and his colleagues study the cellular and molecular mechanisms which control the development function and evolution of commissural neurons which interconnect the two halves of the nervous system. His team also studies the role of axon guidance molecules in retinal neo-angiogenesis, optic nerve regeneration and cornea innervation. In the past few years they have used novel molecular and imaging techniques (such as tissue clearing an 3D light sheet microscopy) to study axon guidance and embryology and they have started to build the first 3D cellular atlas of the developing human embryo. 